\title{
Alimentação na pandemia- como esta questão afetou a saúde bucal infantil - revisão narrativa da literatura
}

\author{
Food in the pandemic- how this issue affected children's oral health - a narrative review of the
} literature

La alimentación en la pandemia - cómo afectó a la salud bucodental de los niños - una revisión narrativa de la literatura

Recebido: 16/09/2021 | Revisado: 22/09/2021 | Aceito: 26/09/2021 | Publicado: 26/09/2021

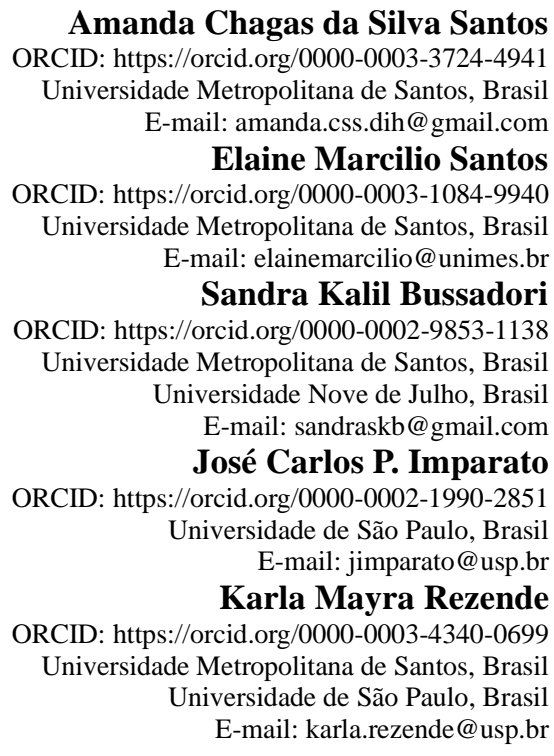

\section{Resumo}

A pandemia do novo coronavírus resultou, desde março de 2020, um grande impacto na vida social, psíquica e emocional. A quarentena, caracterizada pelo isolamento social, despertou ansiedade, pânico e solidão em diversas pessoas. A brusca mudança de hábitos diários como atividades físicas, trabalho e estudo desencadearam sentimentos negativos e mudança na rotina da população em geral. Tratando-se dos hábitos alimentares das crianças, estes também sofreram mudanças, uma vez que os alimentos estão disponíveis em casa durante todo o tempo, sem horários regulares e muitas vezes sendo os do tipo ultraprocessados que apresentam aceitação e consumo exagerado além de baixa demanda mastigatória e embalagens práticas e sedutoras, favorecendo o consumo a qualquer hora e local. Desta forma, o objetivo desse estudo foi realizar uma revisão narrativa para verificar se nesses 18 meses de pandemia, houve estudos que possam associar a alteração da alimentação em crianças durante a pandemia e sua relação com saúde bucal. Com recorte temporal, uma busca eletrônica foi realizada nas bases de dados PubMed/MedLine, no período de 01 de janeiro de 2020 até 31 de agosto de 2021. Foram encontrados 23 artigos, no qual apenas 5 estavam incluídos nos critérios preconizado pelos autores. Concluiu-se que, embora nesta revisão não tenha encontrado associação entre a prevalência de lesões de cárie ou outras doenças bucais, a ingestão alimentar e higiene oral foi alterada. Assim, os efeitos dos hábitos alimentares e diminuição dos cuidados com a higiene oral podem ficar mais evidentes nos próximos meses.

Palavras-chave: COVID-19; Comportamento alimentar; Crianças.

\begin{abstract}
Since March 2020, the new coronavirus pandemic has had a major impact on social, psychological, and emotional life. Quarantine, characterized by social isolation, aroused anxiety, panic, and loneliness in many people. The sudden change in daily habits such as physical activities, work, and study triggered negative feelings and changes in the routine of the population in general. Regarding the children's eating habits, these have also undergone changes, since food is available at home at all times, without regular hours and often being the ultra-processed type that presents exaggerated acceptance and consumption, in addition to low masticatory demand and practical and seductive
\end{abstract}


packaging, favoring consumption at any time and place. Thus, the aim of this study was to carry out a narrative review to verify whether, in these 18 months of the pandemic, there were studies that could associate dietary changes in children during the pandemic and its relationship with oral health. With a time frame, an electronic search was performed in the PubMed/MedLine databases, from January 1, 2020, to August 31, 2021. Twenty-three articles were found, of which only 5 were included in the criteria recommended by the authors. It was concluded that, although this review did not find an association between the prevalence of caries lesions or other oral diseases, food intake and oral hygiene were altered. Thus, the effects of eating habits and reduced care with oral hygiene may become more evident in the coming months.

Keywords: COVID-19; Feeding behavior; Child.

\section{Resumen}

Desde marzo de 2020, la nueva pandemia de coronavirus ha tenido un gran impacto en la vida social, psicológica y emocional. La cuarentena, caracterizada por el aislamiento social, despertó ansiedad, pánico y soledad en muchas personas. El cambio repentino de los hábitos cotidianos, como las actividades físicas, el trabajo y el estudio, desencadenó sentimientos negativos y cambios en la rutina de la población en general. En lo que respecta a los hábitos alimenticios de los niños, éstos también han sufrido cambios, ya que los alimentos están disponibles en casa a todas horas, sin horarios regulares y siendo muchas veces del tipo ultraprocesado que presenta una aceptación y consumo exagerados, además de una baja exigencia masticatoria y un envase práctico y seductor, favoreciendo el consumo en cualquier momento y lugar. Así, el objetivo de este estudio fue realizar una revisión narrativa para verificar si, en estos 18 meses de pandemia, había estudios que pudieran asociar los cambios dietéticos en los niños durante la pandemia y su relación con la salud bucal. Con un marco temporal, se realizó una búsqueda electrónica en las bases de datos PubMed/MedLine, desde el 1 de enero de 2020 hasta el 31 de agosto de 2021. Se encontraron 23 artículos, de los cuales sólo 5 fueron incluidos en los criterios recomendados por los autores. Se concluyó que, aunque esta revisión no encontró una asociación entre la prevalencia de las lesiones de caries u otras enfermedades orales, la ingesta de alimentos y la higiene bucal estaban alteradas. Por lo tanto, los efectos de los hábitos alimentarios y el menor cuidado de la higiene bucal pueden hacerse más evidentes en los próximos meses.

Palabras clave: COVID-19; Conducta alimentaria; Niños.

\section{Introdução}

A Organização Mundial da Saúde anunciou ao mundo em 11 de março de 2020 uma nova pandemia. A Síndrome Respiratória Aguda Grave Coronavírus 2 [SARS-CoV-2], é a mais recente forma de coronavírus que desencadeou um estado de emergência mundial, causando um forte impacto no estilo de vida e, consequentemente, nos hábitos alimentares (Brooks et al., 2020; Carvalho et al., 2020; Huang et al., 2020). Essa nova situação de quarentena em crianças, despertou também sentimentos como ansiedade, tédio, mudanças de humor e solidão (pela falta das atividades escolares e físicas, da convivência social com colegas das escolas e dos pais, que mesmo estando presentes em suas residências, estavam, porém, ocupados com os trabalhos online em casa.

A suspensão de programas escolares assim como as atividades esportivas, a nova rotina adotada por crianças e adolescentes implicou nas mudanças de sono, lazer, saúde física e mental e no comportamento alimentar, uma vez que a criança passou a ter acesso a alimentos à disposição e sem horários regulares (Di Renzo et al., 2020). Os alimentos disponíveis em casa muitas vezes passaram a servir de conforto e companhia, sendo os do tipo ultraprocessados (associados a altos teores de gordura + açúcar ou gordura + sal), apresentam aceitação e consumo exagerado além de baixa demanda mastigatória e embalagens práticas e sedutoras, que favorecem o consumo a qualquer hora e local (Finkelstein-Fox et al., 2020; Ulrich-Lai et al., 2015).

É reconhecido que as doenças bucais compartilham riscos comportamentais comuns com outras principais doenças crônicas não transmissíveis como sobrepeso, obesidade e diabetes tipo 2 (Sheiham \& Watt, 2000).

Desta forma, o objetivo desse estudo foi realizar uma revisão narrativa para verificar se nesses 18 meses de pandemia, houve estudos que possam associar a alteração da alimentação em crianças durante a pandemia e sua relação com saúde bucal. 


\section{Metodologia}

O estudo trata-se de uma revisão de literatura realizada por meio da busca de artigos na base de dados Pubmed/ Medline (PubMed)(Estrela, 2018; Ludke \& Andre, 2013). Os descritores foram escolhidos por sua importância na delimitação do tema de acordo com termos do Medical Subject Headings (MeSH): preschoolers, dental caries, food consumption, Coronavirus or COVID-19, e Eating habits.

Com recorte temporal, uma busca eletrônica foi realizada nas bases de dados PubMed/MedLine, no período de 01 de janeiro de 2020 até 31 de agosto de 2021, para artigos em inglês. No modo "pesquisa avançada", foram utilizados os termos (MeSH) e os Entry Terms combinados com os termos booleanos "OR" e "AND". E as palavras utilizadas foram: Children or Preschool; Pandemics; COVID-19, comfort food, eating habits, dental caries, oral healthy.

Os dados foram selecionados de acordo com os seguintes critérios: trabalhos completos em inglês e que estivessem de acordo com a narrativa do tema, ou seja, mudança da dieta da criança durante a pandemia e sua interferência na saúde bucal. Para a escolha foram considerados os seguintes tipos de artigos: revisão de literatura, revisão sistemática, casos clínicos e trabalhos de pesquisas clinicas de estudos clínicos, longitudinais e ou comportamental.

Os processos de busca, triagem e coleta de dados foram conduzidos por duas autoras. Na primeira etapa os títulos e resumos foram verificados e excluídos os registros que estavam fora do tema, ou seja, tipos e trabalhos não relacionados à alimentação em criança. Desacordos entre os autores, foram resolvidos em discussões detalhadas com a orientadora. Os dados coletados de cada artigo incluído foram: ano, título, idade das crianças, tipo de doenças bucais e conclusão. Os dados foram analisados de maneira descritiva.

\section{Resultados e Discussão}

Foram encontrados 23 artigos, no qual apenas 5 estavam incluídos nos critérios preconizado pelos autores. As principais informações contidas em cada artigo encontram-se descritas no Quadro 1 e ordenadas de acordo com o ano de publicação. 
Research, Society and Development, v. 10, n. 12, e461101220679, 2021

(CC BY 4.0) | ISSN 2525-3409 | DOI: http://dx.doi.org/10.33448/rsd-v10i12.20679

\begin{tabular}{|c|c|c|c|c|c|c|}
\hline & Ano & Autores & Título dos artigos & $\begin{array}{l}\text { Idade das } \\
\text { crianças }\end{array}$ & Tipo de doença bucal & Conclusão. \\
\hline 1 & 2020 & Chan et al. & $\begin{array}{l}\text { A systematic review of dietary acids and habits } \\
\text { on dental erosion in adolescents }\end{array}$ & 10 a 19 & Erosão dentária & $\begin{array}{l}\text { Sugere que certos fatores de risco } \\
\text { dietéticos podem contribuir para a erosão } \\
\text { dentária em adolescentes. }\end{array}$ \\
\hline 2 & 2020 & Olczak-Kowalczyk & $\begin{array}{l}\text { Factors Associated with Early Childhood Caries } \\
\text { in Polish Three-Year-Old Children }\end{array}$ & 0 a 3 & Cárie na primeira infância & $\begin{array}{l}\text { O consumo de alimentos açucarados nos } \\
\text { primeiros } 2 \text { anos de idade e consumo } \\
\text { noturno de bebidas doces por crianças } \\
\text { maiores de } 12 \text { meses. }\end{array}$ \\
\hline 3 & 2020 & Tudoroniu & $\begin{array}{l}\text { Correlation of Caries Prevalence, Oral Health } \\
\text { Behavior and Sweets Nutritional Habits among } \\
10 \text { to } 19 \text {-Year-Old Cluj-Napoca Romanian } \\
\text { Adolescents }\end{array}$ & 10 a 19 & Cárie & 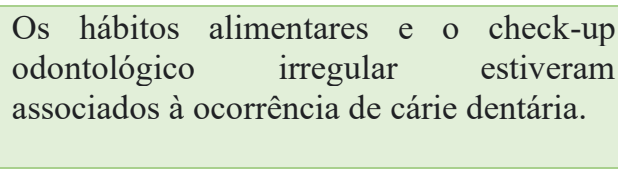 \\
\hline 4 & 2021 & Garcia-Pola & $\begin{array}{l}\text { Promoting oral health among 6-year old } \\
\text { children: The impact of social environment and } \\
\text { feeding behavior }\end{array}$ & 6 & Carie & $\begin{array}{l}\text { Consumo de alimentos açucarados e ao } \\
\text { excesso de peso. }\end{array}$ \\
\hline 5 & 2021 & Docimo & $\begin{array}{l}\text { Cariogenic Risk and COVID-19 Lockdown in a } \\
\text { Paediatric Population }\end{array}$ & 4 a 14 & Cárie & $\begin{array}{l}\text { Aumento risco de cárie durante a } \\
\text { pandemia. }\end{array}$ \\
\hline
\end{tabular}

Fonte: Autores. 
Fazendo um panorama dos artigos publicados, pelo fato da utilizar uma base de dados internacional, os trabalhos aqui selecionados e mostrado, no Quadro 1, eram da Austrália, Polônia, Romênia, Espanha e Itália.

O primeiro trabalho foi uma revisão sistemática, publicada no ano de 2020 com desfecho na erosão dentária (Chan et al., 2020). Nos trabalhos sobre a prevalência de cárie, seja na primeira infância ou crianças e adolescentes, os estudos eram do tipo transversal e observacional (Garcia-Pola et al., 2021; Olczak-Kowalczyk et al., 2020; Tudoroniu et al., 2020) no qual houveram uma associação da prevalência de cárie em crianças que tinham uma dieta cariogênica e sem visitas regulares no dentista. Apesar destes artigos estarem de acordo com os critérios de inclusão sobre alimentação e doença bucal, eles possivelmente foram realizados antes da pandemia e publicados nos anos de 2020 e 2021. Apenas um artigo, realizado por meio de questionário online, foi especifico sobre o risco de alimentação cariogênica durante o isolamento social (Docimo et al., 2021). Neste trabalho os autores fizeram, por meio de teleconsulta, um questionário aos pais e responsáveis sobre a mudança de alimentação durante a pandemia do COVID-19. Os autores concluíram que neste período de isolamento social, houve um aumento na ingestão de açúcares fermentáveis, consumo frequente de açúcares dietéticos, ingestão frequente de lanches entre as refeições. No quesito higiene bucal, este também ficou deficiente na frequência, tempo de escovação e uso de dentifrício com concentração de flúor adequado. De modo interessante, esse trabalho só foi possível graças ao auxílio da teleodontologia e o telemonitoramento, ferramenta esta, que se tornou essencial como alternativa na forma de auxilio, acompanhamento e orientação em diversos casos relacionados aos cuidados da saúde bucal (Machado et al., 2021; Revista de Odontopediatría Latinoamericana, 2021; Silva et al., 2021). Isso porque até a metade do ano de 2020, as consultas odontológicas também se tornaram restritas com atendimentos nos casos de urgências e ou emergências (Credie et al., 2020; Paglia, 2020).

A presença das crianças em casa gerou uma necessidade de reorganização da dinâmica familiar. Nesse contexto, houve uma mudança na rotina, pois, repentinamente, houve cancelamentos das aulas, atividades extracurriculares, esportes, lazer e convívio entre os amigos e familiares. Os impactos do COVID-19 na segurança alimentar e nutricional pode ter acontecido por dois fatores, o primeiro seria pela dificuldade financeira vivenciadas em suas famílias, uma vez que muitas pessoas perderam seu emprego neste período. Concomitantemente, há evidência de que as alterações de hábitos alimentares, fácil acesso aos alimentos, ansiedades, falta de horários para se alimentares e ausência das atividades físicas diárias são tidas como principais responsáveis pelo aumento da incidência da obesidade (Ataey et al., 2020). Desemprego, diminuição ou corte da renda, indicaram um aumento do consumo de alimentos industrializados com um alto teor energético, contendo carboidratos simples e gorduras saturadas, e a baixa concentração de vitaminas e minerais pelo valor econômico ser mais baixo e de fácil acesso (Lima et al., 2021; Lustig, 2020). O segundo fator, é justificar na alimentação um momento de conforto e segurança no meio da insegurança da pandemia. Os chamados "comfort food", ou comida conforto, que se tornou uma tendência em alimentação moderna, com o objetivo de despertar emoções através do paladar, com pratos que remetam a momentos especiais e como forma de recompensa (Ammar et al., 2020; Herhaus et al., 2020). A diretriz da Organização Mundial de Saúde orientar a diminuir o consumo de açúcar. E essa diretriz pode ser uma oportunidade e um apoio para odontologia em seu objetivo de levar ao público a mensagem de orientação sobre os malefícios desses alimentos na saúde geral e bucal. Os fatores relacionados aos alimentos envolvem a liberação de açúcares, a viscosidade do produto, e o tipo e a concentração do açúcar. Já os fatores relacionados ao consumo, está diretamente sobre a questão da frequência do consumo, dos hábitos de beber e mastigar, da eficiência da mastigação e da deglutição, do fluxo e composição salivar, da presença de biofilme cariogênico e o uso de fluoretos. É uma observação comum que, com o número comparável de produtos contendo açúcar, algumas pessoas são capazes de controlar o risco e não desenvolverão cárie, enquanto outras desenvolverão quantidades significativas de cárie dentária.(van Loveren, 2019).

Por fim, dos estudos relacionados a essa temática proposta, podemos concluir que a pandemia causou efeitos psicológicos nas crianças, que estão de alguma forma ligados às mudanças nos padrões de ingestão alimentar e na rotina de 
higiene oral. Embora nesta revisão não tenha encontrado associação entre a prevalência de lesões de cárie, ingestão alimentar e higiene, deve-se considerar que o período de análise dos artigos publicados está limitado uma vez que o estágio é relativamente inicial da pandemia. Assim, os efeitos dos hábitos alimentares e diminuição dos cuidados com a higiene oral podem ficar mais evidentes nos próximos meses. Deixamos aqui em aberto para novas pesquisas sobre essa temática para realização de estudos clínicos longitudinais que possam associar o impacto da pandemia na mudança da alimentação e doenças bucais em crianças, uma vez que os consultórios, escolas e atividades estão retornando de forma gradual e cuidadosa.

\section{Conclusão}

Concluiu-se que, neste período pandêmico, houve alteração da alimentação e rotina da criança porém sem associação se houve um impacto na prevalência bucal. O uso de tecnologias de comunicação é uma alternativa para realizar programa de prevenção à doenças bucais dirigido a pais como forma de interceptar fatores de risco de doenças bucais e estimular comportamentos de proteção, cuidado e higiene bucal, considerando também o prolongamento do período de pandemia.

\section{Referências}

Ammar, A., Brach, M., Trabelsi, K., Chtourou, H., Boukhris, O., Masmoudi, L., Bouaziz, B., Bentlage, E., How, D., Ahmed, M., Muller, P., Muller, N., Aloui, A., Hammouda, O., Paineiras-Domingos, L. L., Braakman-Jansen, A., Wrede, C., Bastoni, S., Pernambuco, C. S., Mataruna, L., Taheri, M., Irandoust, K., Khacharem, A., Bragazzi, N. L., Chamari, K., Glenn, J. M., Bott, N. T., Gargouri, F., Chaari, L., Batatia, H., Ali, G. M., Abdelkarim, O., Jarraya, M., Abed, K. E., Souissi, N., Van Gemert-Pijnen, L., Riemann, B. L., Riemann, L., Moalla, W., Gomez-Raja, J., Epstein, M., Sanderman, R., Schulz, S. V., Jerg, A., AlHorani, R., Mansi, T., Jmail, M., Barbosa, F., Ferreira-Santos, F., Simunic, B., Pisot, R., Gaggioli, A., Bailey, S. J., Steinacker, J. M., Driss, T., \& Hoekelmann, A. (2020). Effects of COVID-19 Home Confinement on Eating Behaviour and Physical Activity: Results of the ECLB-COVID19 International Online Survey. Nutrients, 12(6). https://doi.org/10.3390/nu12061583

Ataey, A., Jafarvand, E., Adham, D., \& Moradi-Asl, E. (2020). The Relationship Between Obesity, Overweight, and the Human Development Index in World Health Organization Eastern Mediterranean Region Countries. J Prev Med Public Health, 53(2), 98-105. https://doi.org/10.3961/jpmph.19.100

Brooks, S. K., Webster, R. K., Smith, L. E., Woodland, L., Wessely, S., Greenberg, N., \& Rubin, G. J. (2020). The psychological impact of quarantine and how to reduce it: rapid review of the evidence. Lancet, 395(10227), 912-920. https://doi.org/10.1016/S0140-6736(20)30460-8

Carvalho, P. M. M., Moreira, M. M., de Oliveira, M. N. A., Landim, J. M. M., \& Neto, M. L. R. (2020). The psychiatric impact of the novel coronavirus outbreak. Psychiatry Res, 286, 112902. https://doi.org/10.1016/j.psychres.2020.112902

Chan, A. S., Tran, T. T. K., Hsu, Y. H., Liu, S. Y. S., \& Kroon, J. (2020). A systematic review of dietary acids and habits on dental erosion in adolescents. Int J Paediatr Dent, 30(6), 713-733. https://doi.org/10.1111/ipd.12643

Credie, G. B., Coelho, A. A., \& Rezende, K. M. (2020). Coronavirus (COVID-19) in children: history and pediatric oral health. Dental Oral biology and craniofacial research, 3(3), 3. https://doi.org/http://dx.doi.org/10.31487/j.DOBCR.2020.03.06

Di Renzo, L., Gualtieri, P., Pivari, F., Soldati, L., Attina, A., Cinelli, G., Leggeri, C., Caparello, G., Barrea, L., Scerbo, F., Esposito, E., \& De Lorenzo, A. (2020). Eating habits and lifestyle changes during COVID-19 lockdown: an Italian survey. J Transl Med, 18(1), 229. https://doi.org/10.1186/s12967-020$02399-5$

Docimo, R., Costacurta, M., Gualtieri, P., Pujia, A., Leggeri, C., Attina, A., Cinelli, G., Giannattasio, S., Rampello, T., \& Di Renzo, L. (2021). Cariogenic Risk and COVID-19 Lockdown in a Paediatric Population. Int J Environ Res Public Health, 18(14). https://doi.org/10.3390/ijerph18147558

Estrela, C. (2018). Metodologia cientifica: ciênica, ensino, pesquisa (A. Médicas, Ed.).

Finkelstein-Fox, L., Gnall, K. E., \& Park, C. L. (2020). Mindfulness moderates daily stress and comfort food snacking linkages: a multilevel examination. $J$ Behav Med, 43(6), 1062-1068. https://doi.org/10.1007/s10865-020-00164-z

Garcia-Pola, M., Gonzalez-Diaz, A., \& Garcia-Martin, J. (2021). Promoting oral health among 6-year old children: The impact of social environment and feeding behavior. Community Dent Health, 38(2), 76-82. https://doi.org/10.1922/CDH_00065Garcia-Pola07

Herhaus, B., Ullmann, E., Chrousos, G., \& Petrowski, K. (2020). High/low cortisol reactivity and food intake in people with obesity and healthy weight. Transl Psychiatry, 10(1), 40. https://doi.org/10.1038/s41398-020-0729-6

Huang, C., Wang, Y., Li, X., Ren, L., Zhao, J., Hu, Y., Zhang, L., Fan, G., Xu, J., Gu, X., Cheng, Z., Yu, T., Xia, J., Wei, Y., Wu, W., Xie, X., Yin, W., Li, H., Liu, M., Xiao, Y., Gao, H., Guo, L., Xie, J., Wang, G., Jiang, R., Gao, Z., Jin, Q., Wang, J., \& Cao, B. (2020). Clinical features of patients infected with 2019 novel coronavirus in Wuhan, China. Lancet, 395(10223), 497-506. https://doi.org/10.1016/S0140-6736(20)30183-5

Lima, E. R., Silva, T. S. S., Vilela, A. B. A., Rodrigues, V. P., \& Boery, T. N. S. d. O. (2021). Implications of the COVID-19 pandemic in brazilian food habits: integrative review. Research, Society and Development, 10(4), 10. https://doi.org/10.33448/rsd-v10i4.14125

Ludke, M., \& Andre, M. E. D. A. (2013). Pesquisas em educação: uma abordagem qualitativa. (E.P.U., Ed.). 
Research, Society and Development, v. 10, n. 12, e461101220679, 2021 (CC BY 4.0) | ISSN 2525-3409 | DOI: http://dx.doi.org/10.33448/rsd-v10i12.20679

Lustig, R. H. (2020). Ultraprocessed Food: Addictive, Toxic, and Ready for Regulation. Nutrients, 12(11). https://doi.org/10.3390/nu12113401

Machado, F. C., Oliveira, L. C., Silva, D. L. M., Novais, V. R., \& Menezes, M. d. S. (2021). Teleorientation with the use of digital tools to assist dental care in a time of the COVID-19 pandemic: an integrative literature review Research, Society and Development, 10(6), 9. https://doi.org/10.33448/rsd-v10i6.15663

Olczak-Kowalczyk, D., Gozdowski, D., \& Kaczmarek, U. (2020). Factors Associated with Early Childhood Caries in Polish Three-Year-Old Children. Oral Health Prev Dent, 18(1), 833-842. https://doi.org/10.3290/j.ohpd.a45088

Paglia, L. (2020). COVID-19 and Paediatric Dentistry after the lockdown. Eur J Paediatr Dent, 21(2), 89. https://doi.org/10.23804/ejpd.2020.21.02.01

Revista de Odontopediatría Latinoamericana, E. d. t. m. d. 1. (2021). Teleodontologia: Aplicação à Odontopediatria durante a pandemia COVID-19. Journal of Latin American Pediatric Dentistry, 10(2), 20. https://doi.org/https://doi.org/10.47990/alop.v10i2.192

Sheiham, A., \& Watt, R. G. (2000). The common risk factor approach: a rational basis for promoting oral health. Community Dent Oral Epidemiol, 28(6), 399406. https://doi.org/10.1034/j.1600-0528.2000.028006399.x

Silva, R. V., Imparato, J. C. P., Bussadori, S. K., Santos, E. M., \& Rezende, K. M. (2021). Telemonitoring and spontaneous resolution in delayed eruption of central incisors during tge pandemic - Case report. Research, Society and Development, 10(7), 6. https://doi.org/https://doi.org/10.33448/rsd-v10i7.16439

Tudoroniu, C., Popa, M., Iacob, S. M., Pop, A. L., \& Nasui, B. A. (2020). Correlation of Caries Prevalence, Oral Health Behavior and Sweets Nutritional Habits among 10 to 19-Year-Old Cluj-Napoca Romanian Adolescents. Int J Environ Res Public Health, 17(18). https://doi.org/10.3390/ijerph17186923

Ulrich-Lai, Y. M., Fulton, S., Wilson, M., Petrovich, G., \& Rinaman, L. (2015). Stress exposure, food intake and emotional state. Stress, 18(4), 381-399. https://doi.org/10.3109/10253890.2015.1062981

van Loveren, C. (2019). Sugar Restriction for Caries Prevention: Amount and Frequency. Which Is More Important? Caries Res, 53(2), 168-175. https://doi.org/10.1159/000489571 